\title{
The nearly invisible intraneural cyst: a new and emerging part of the spectrum
}

\author{
Thomas J. Wilson, MD, ${ }^{1}$ Marie-Noëlle Hébert-Blouin, MD, ${ }^{2}$ Naveen S. Murthy, MD, ${ }^{3}$ \\ Joaquín J. García, MD, ${ }^{4}$ Kimberly K. Amrami, MD, ${ }^{3}$ and Robert J. Spinner, MD ${ }^{1}$
}

Departments of ${ }^{1}$ Neurosurgery, ${ }^{3}$ Radiology, and ${ }^{4}$ Laboratory Medicine and Pathology, Mayo Clinic, Rochester, Minnesota; and ${ }^{2}$ Department of Neurosurgery, McGill University Health Centre, Montreal, Quebec, Canada

\begin{abstract}
OBJECTIVE The authors have observed that a subset of patients referred for evaluation of peroneal neuropathy with "negative" findings on MRI of the knee have subtle evidence of a peroneal intraneural ganglion cyst on subsequent closer inspection. The objective of this study was to introduce the nearly invisible peroneal intraneural ganglion cyst and provide illustrative cases. The authors further wanted to identify clues to the presence of a nearly invisible cyst.

METHODS Illustrative cases demonstrating nearly invisible peroneal intraneural ganglion cysts were retrospectively reviewed and are presented. Case history and physical examination, imaging, and intraoperative findings were reviewed for each case. The outcomes of interest were the size and configuration of peroneal intraneural ganglion cysts over time, relative to various interventions that were performed, and in relation to physical examination and electrodiagnostic findings.

RESULTS The authors present a series of cases that highlight the dynamic nature of peroneal intraneural ganglion cysts and introduce the nearly invisible cyst as a new and emerging part of the spectrum. The cases demonstrate changes in size and morphology over time of both the intraneural and extraneural compartments of these cysts. Despite "negative" MR imaging findings, nearly invisible cysts can be identified in a subset of patients.

CONCLUSIONS The authors demonstrate here that peroneal intraneural ganglion cysts ride a roller coaster of change in both size and morphology over time, and they describe the nearly invisible cyst as one end of the spectrum. They identified clues to the presence of a nearly invisible cyst, including deep peroneal predominant symptoms, fluctuating symptoms, denervation changes in the tibialis anterior muscle, and abnormalities of the superior tibiofibular joint, and they correlate the subtle imaging findings to the internal fascicular topography of the common peroneal nerve. The description of the nearly invisible cyst may allow for increased recognition of this pathological entity that occurs with a spectrum of findings.

https://thejns.org/doi/abs/10.3171/2016.12.FOCUS16439
\end{abstract}

KEY WORDS peroneal nerve; ganglion cyst; intraneural; articular branch; superior tibiofibular joint

$\mathrm{W}$ HILE intraneural ganglion cysts can be associated with any joint, peroneal cysts associated with the superior tibiofibular joint (STFJ) are the most common. Intraneural cysts form from synovial joints, via a capsular defect, as synovial fluid dissects along the articular branch toward the parent nerve., ${ }^{1,3,-11,14-17,20}$ The extent and dimensions of intraneural cysts are determined by the path of least resistance, intraarticular pressure, and pressure fluxes. In some cases, pressure can drive extreme longitudinal propagation..$^{18}$ For peroneal intraneural cysts, these principles may lead to phasic propagation, including primary ascent of cyst fluid up the articular branch and common peroneal nerve, cross-over within the sciatic nerve, and terminal branch descent down the tibial nerve. ${ }^{13}$

In contrast, we have evaluated several patients with peroneal neuropathy and "negative" MRI findings who, on 
subsequent closer inspection, have had subtle evidence of an intraneural ganglion cyst. We present a series of cases that highlight the dynamic nature of peroneal intraneural ganglion cysts and describe the nearly invisible cyst as a new and emerging part of the spectrum.

\section{Methods \\ Patient Cohort}

Cases in which nearly invisible peroneal intraneural ganglion cysts were found were retrospectively reviewed and are presented. Case history and physical examination, imaging, and intraoperative findings were reviewed for each case. This study was approved by the Institutional Review Board of the Mayo Clinic.

\section{Variables of Interest}

Data abstracted included neurological examination, electrodiagnostic, and imaging findings; available ultrasound images, MR images, and MR arthrograms were reviewed for abnormalities of the common peroneal nerve and its branches as well as abnormalities of the STFJ.

\section{Outcomes of Interest}

The outcomes of interest were the size and configuration of peroneal intraneural ganglion cysts over time, relative to various interventions that were performed, and in relation to physical examination and electrodiagnostic findings.

\section{Results}

\section{Case 1: Sequential MR Images Demonstrate a Shrinking Cyst}

Approximately 3 months prior to our evaluation, a 41-year-old man developed the acute onset of left lateral knee pain and a partial foot drop while performing squatting exercises that progressed to a complete foot drop over several months. Electromyography (EMG) revealed a deep predominant peroneal neuropathy superimposed on an L-5 radiculopathy. MRI showed an extreme peroneal intraneural ganglion cyst, which arose from the STFJ and extended to the sciatic nerve bifurcation (Fig. 1A and B). Because MRI did not capture the full extent of the cyst, 19 days later repeat MRI with a larger field was performed. The repeat MRI study showed a substantially smaller peroneal intraneural ganglion cyst (Fig. 1C and D). At the time of our evaluation, the patient had absent tibialis anterior muscle function with normal peroneus muscle function, suggesting a deep peroneal predominant neuropathy. At surgery, the articular branch and the joint surfaces of the STFJ were resected. The common peroneal nerve and the articular branch were clearly cystic (Fig. 1E). Histopathological examination of the articular branch was consistent with an intraneural ganglion cyst (Fig. 1F). At the 6-month follow-up, the patient had Medical Research Council Grade 4-/5 dorsiflexion and normal eversion. At the most recent follow-up, 2.5 years after the operation, he had only trace weakness in his tibialis anterior muscle, did not require an ankle-foot orthosis, and continued to have decreased sensation in the first dorsal web space.
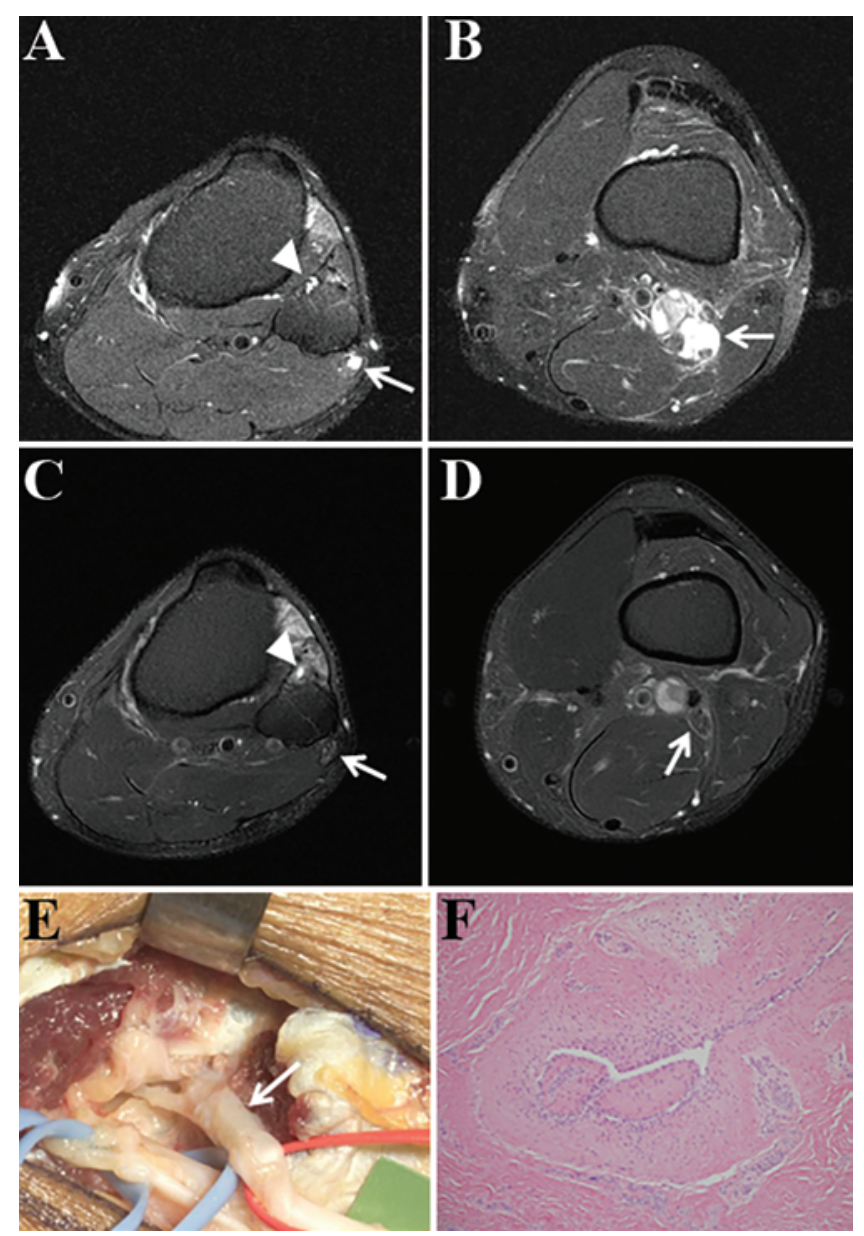

FIG. 1. Case 1. A: Axial T2-weighted image with fat suppression at the level of the fibular head, showing classic findings of an peroneal intraneural ganglion cyst including the signet ring sign (arrow) and tail sign (arrowhead). B: Axial T2-weighted image with fat suppression at the level of the distal femur showing cyst within the sciatic nerve (arrow). C and D: Images obtained 19 days later. Subsequent axial T2-weighted image with fat suppression at the level of the fibular head, showing only subtle findings of an intraneural cyst, including a subtle tail sign (arrowhead) and crescentic T2 hyperintensity around the common peroneal nerve (arrow, C). Subsequent axial T2-weighted image with fat suppression at the level of the distal femur, showing near normalization of the sciatic nerve, with only trace circumferential T2 hyperintensity (wedding ring sign, D). E: Intraoperative photograph showing the trifurcation of the common peroneal nerve with a cystic articular branch (arrow). F: Low-power photomicrograph revealing a pseudocyst surrounded by a wall of dense fibrosis, features characteristic of a ganglion cyst. No inflammation was identified. $\mathrm{H} \& \mathrm{E}$, original magnification $\times 200$.

\section{Case 2: Ultrasonography Followed by MRI Demonstrates a Shrinking Cyst}

A 33-year-old woman developed an acute, painless foot drop with no clear inciting event. EMG findings were consistent with a severe common peroneal neuropathy. An ultrasound image of the common peroneal nerve was then obtained, which revealed an intraneural cyst (Fig. 2A). On our initial examination approximately 3 months after the onset of the patient's symptoms, the patient had only trace activation of the tibialis anterior and peroneus muscles. MRI and MR arthrography were performed, which re- 


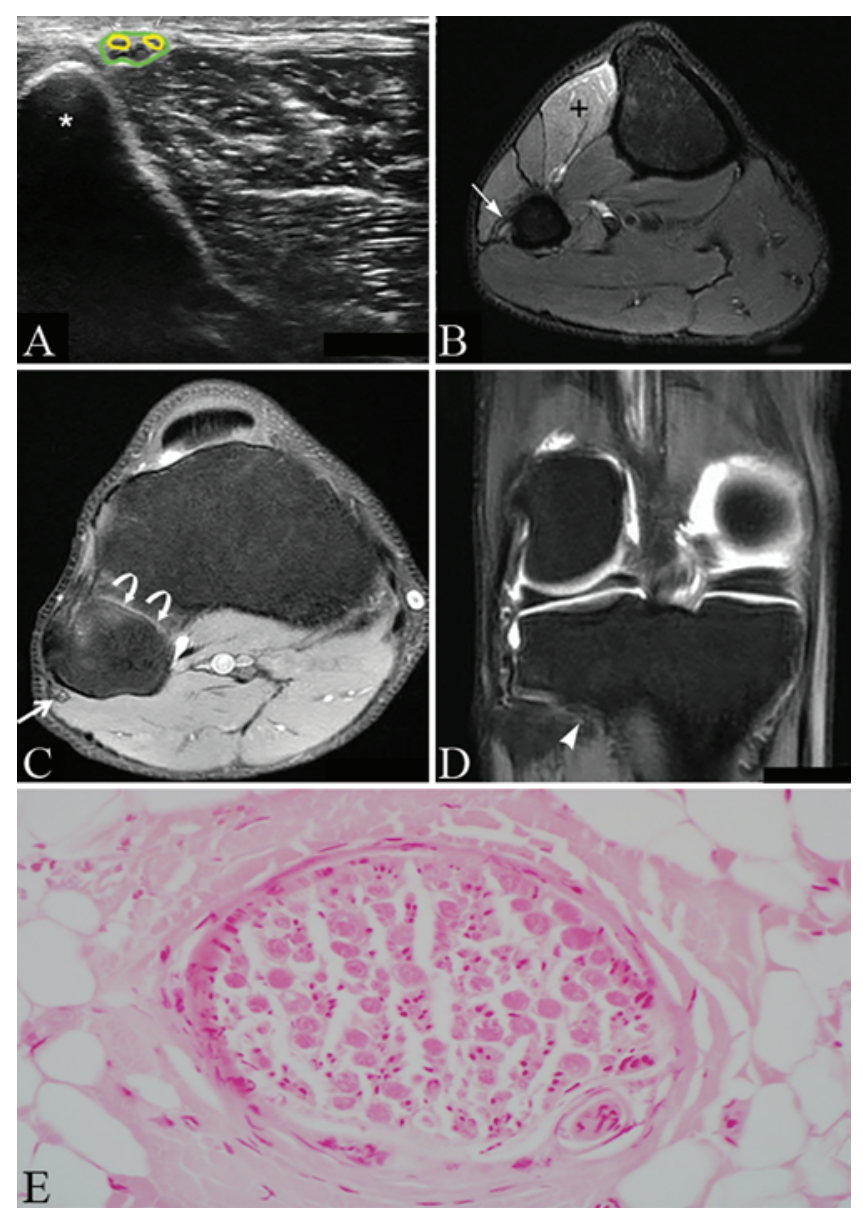

FIG. 2. Case 2. A: High-resolution transverse ultrasound image at the level of the fibular head (asterisk), showing a hypoechoic cyst (yellow outline) within the common peroneal nerve (green outline). B: Axial T2-weighted image with fat suppression at the level of the fibular neck obtained 5 weeks later, showing denervation changes involving the tibialis anterior muscle (cross) and very subtle linear-appearing cyst within the articular branch of the common peroneal nerve (arrow). C: Axial T1-weighted image with fat suppression after direct gadolinium arthrography at the level of the superior tibiofibular joint obtained 5 weeks later, showing subtle contrast within the intraneural cyst at the fibular head (arrow). Note contrast within the STFJ (curved arrows). D: Coronal T2weighted image with fat suppression obtained after arthrography, showing the joint connection from the STFJ to the cyst, with contrast in the articular branch (arrowhead). E: Low-power photomicrograph showing the peripheral nerve with no significant pathological abnormality. Turnbull's blue stain, original magnification $\times 400$.

vealed subtle T2 signal in the peroneal nerve and gadolinium within the anterior portion of the peroneal nerve following intraarticular injection, consistent with a possible small intraneural cyst (Fig. 2B-D); this was in marked contrast to the definite intraneural cyst that was observed on ultrasonography. At surgery, the common peroneal nerve was neurolyzed at the fibular neck, and a cysticappearing articular branch was resected. The STFJ was not resected. Histopathological analysis did not reveal an intraneural cyst (Fig. 2E). At the 6-month follow-up, the patient had normal dorsiflexion and eversion, which continued through the most recent follow-up, approximately 2 years after her operation.

\section{Case 3: Utilizing the Internal Topography of the Common Peroneal Nerve}

A 69-year-old man experienced the insidious onset of a partial right foot drop, which progressed to a complete foot drop over several months. Ultrasonography showed an intraneural peroneal cyst (Fig. 3A and B). On our evaluation, the patient had minimal activation of the tibialis anterior muscle with near-complete preservation of peroneus muscle function. Four months after the ultrasound study, an MRI study was obtained and showed subtle signs of cyst within the common peroneal nerve and articular branch but appreciably smaller than seen on the previous ultrasound study (Fig. 3C and D). At surgery, the common peroneal nerve was neurolyzed at the fibular neck, and both the articular branch and STFJ were resected. By 1 month postoperatively, the patient had only trace weakness of dorsiflexion and was no longer requiring an anklefoot orthosis.

\section{Case 4: Intraosseous Cyst: Clue to an Intraneural Cyst?}

A 45-year-old man presented with a history of intermittent foot drop with multiple occurrences over the previous 7 years. One month prior to presentation, the patient had an episode of foot drop, but this had largely resolved by the time of our evaluation. EMG revealed evidence of a deep predominant peroneal neuropathy. Ultrasonography performed at the time of EMG reportedly showed a possible small intraneural cyst. Because of the suggestion of a small cyst on ultrasonography, an MRI/MR arthrogram was obtained. While most of the intraarticular gadolinium leaked from a ruptured popliteal cyst into the space surrounding the semimembranosus muscle (Fig. 4A), some
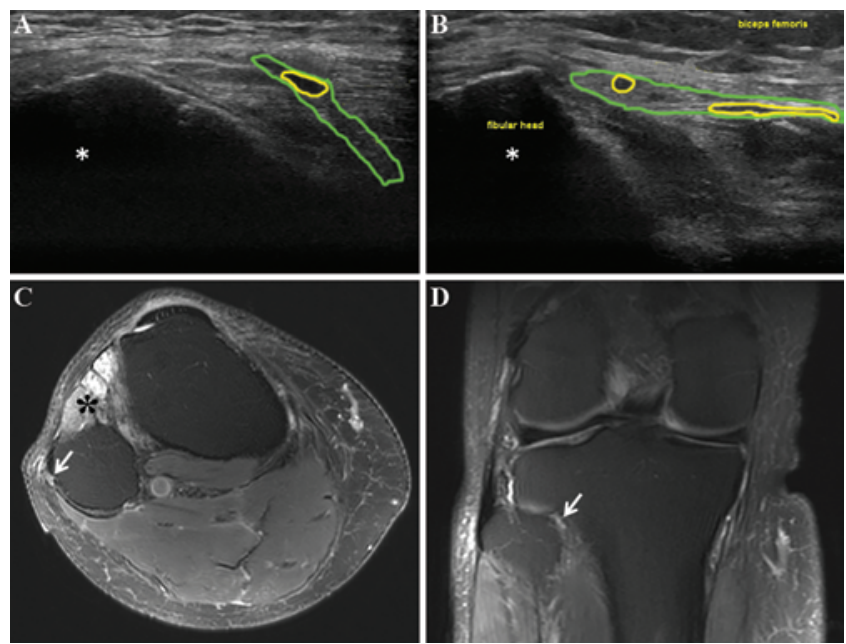

FIG. 3. Case 3. A. Longitudinal ultrasound image obtained at an outside hospital of the common peroneal nerve at the fibular head (asterisk), showing cyst (yellow outline) within the articular branch (green outline). B: Transverse ultrasound image at the fibular head (asterisk) showing the cyst (yellow outline) within the common peroneal nerve (green outline). C: Axial T2-weighted image with fat suppression at the level of the fibular head, showing a subtle cyst within the common peroneal nerve (arrow) involving the anterolateral fascicles and denervation changes within the tibialis anterior muscle (asterisk). D: Coronal T2weighted image with fat suppression showing a small cyst (arrow) within the articular branch of the peroneal nerve. 

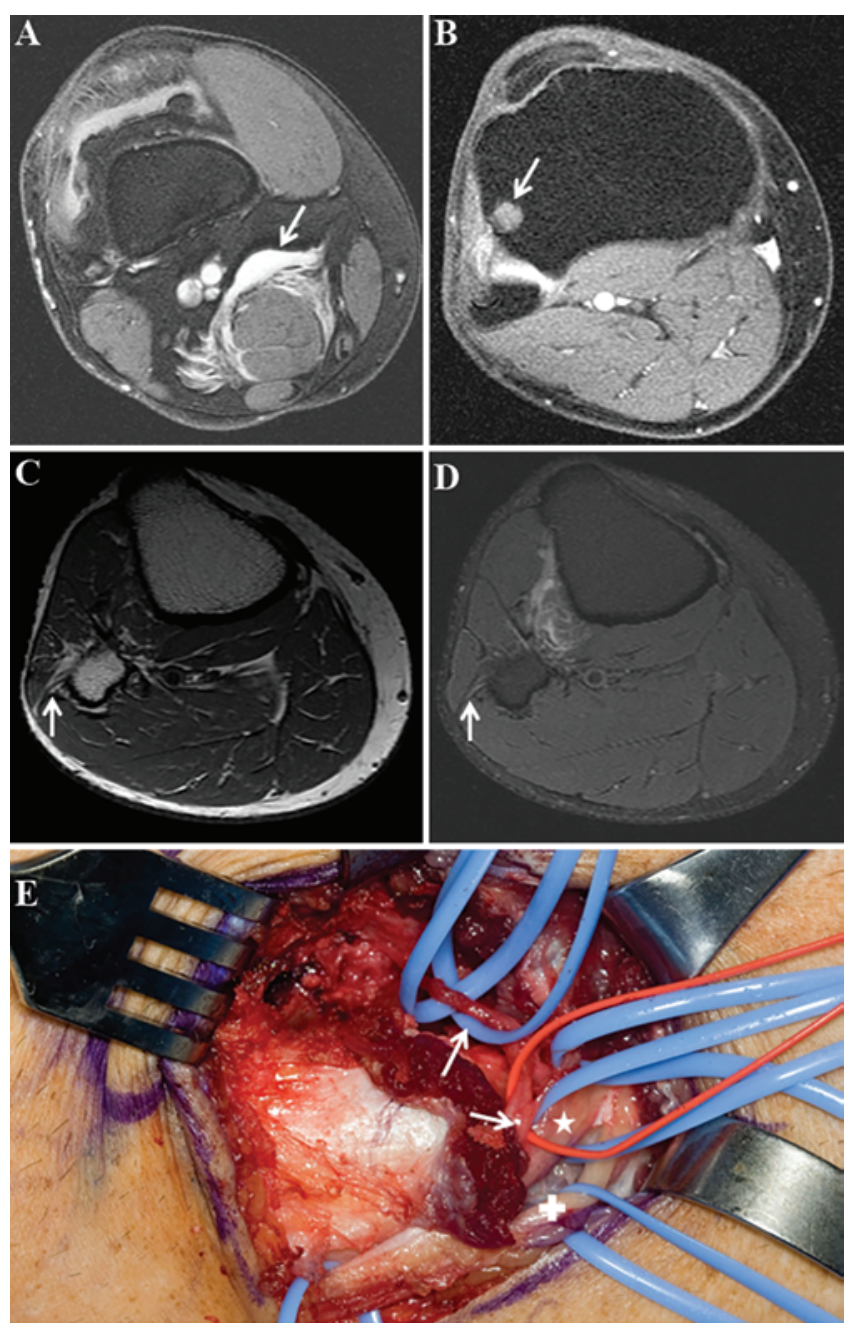

FIG. 4. Case 4. A: Axial T1-weighted image obtained after intraarticular gadolinium injection, showing gadolinium accumulating around the semimembranosus muscle (arrow) after leaking from a ruptured popliteal cyst. B: Axial T1-weighted spoiled gradient recalled echo image with fat suppression after direct gadolinium arthrography but without intravenous contrast, showing an intraosseous ganglion cyst filled with contrast (arrow) within the tibia adjacent to the superior tibiofibular joint. $\mathbf{C}$ and D: Axial T1-weighted (C) and T2-weighted (D) images obtained after intraarticular gadolinium injection, showing a small amount of gadolinium (arrows) within the common peroneal nerve. E: Intraoperative photograph showing the common peroneal nerve through its trifurcation into the superficial peroneal nerve (cross), deep peroneal nerve (star), and articular branch (arrows). The articular branch appeared enlarged and questionably cystic.

of the injected gadolinium filled the intraosseous ganglion cyst, demonstrating the joint connection to the STFJ (Fig. 4B), and a small amount of contrast passed into the common peroneal nerve (Fig. 4C and D), consistent with a small intraneural cyst. The patient underwent decompression of the peroneal nerve and resection of the articular branch and STFJ. The articular branch appeared enlarged and questionably cystic intraoperatively (Fig. 4E). Pathological examination confirmed the presence of intraneural ganglion cyst. One month postoperatively, the patient had normal dorsiflexion and eversion.
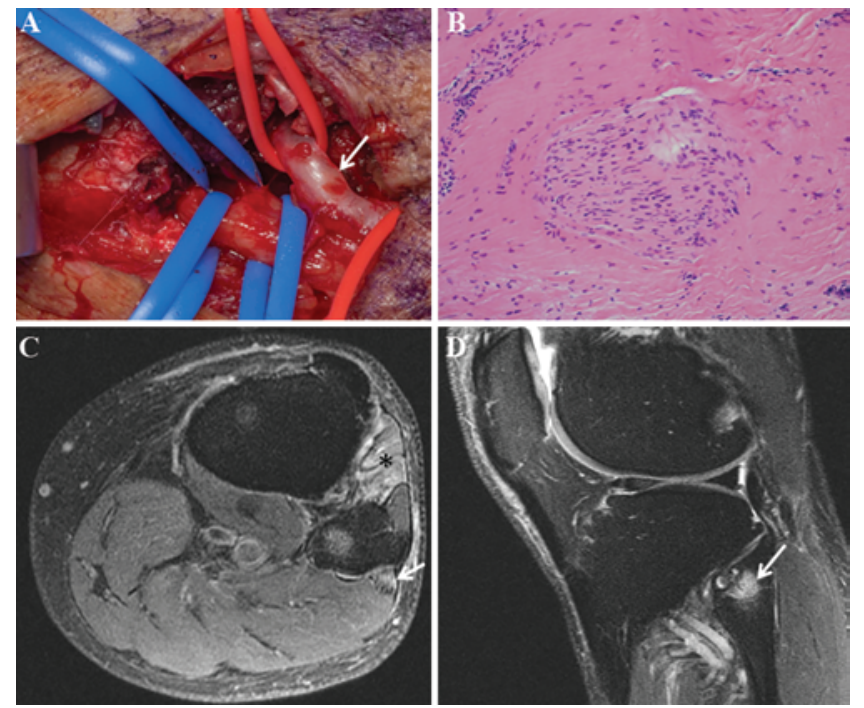

FIG. 5. Case 5. A: Intraoperative photograph showing a cystic, enlarged peroneal articular branch (arrow). B: Low-power photomicrograph revealing peripheral nerve with fibrotic perineurium and reactive changes, features characteristic of an intraneural ganglion cyst. $H$ \& $E$, original magnification $\times 200$. C: Axial T2-weighted image with fat suppression, showing subacute denervation changes in the tibialis anterior muscle (asterisk) and a small cyst within the common peroneal nerve involving the anterolateral fascicles (arrow). D: Sagittal T2-weighted image with fat suppression showing degenerative changes in the STFJ with bone marrow edema in the fibula adjacent to a subchondral cyst (arrow).

\section{Case 5: Abnormal STFJ and Tibialis Anterior Muscle Denervation: Clues to an Intraneural Cyst?}

A 64-year-old man developed the acute onset of right foot drop without any inciting event. On examination, dorsiflexion was $4-/ 5$ and eversion was $5 / 5$. An MRI study revealed degenerative changes in the STFJ with bone marrow edema in the fibular head and subacute denervation in the tibialis anterior muscle, but the images were read as negative for mass or cyst involving the peroneal nerve. Decompression of the common peroneal nerve was planned. With the patient under mild sedation, the common peroneal nerve was noted to be mildly cystic intraoperatively, so decompression was carried further distally and the trifurcation was uncovered. The articular branch was enlarged and cystic (Fig. 5A). The articular branch was dissected distally to the STFJ and resected. No resection of the STFJ was performed. Histological analysis revealed a fibrotic perineurium with reactive changes consistent with an intraneural cyst (Fig. 5B). On subsequent detailed review of the preoperative MRI study, subtle signs of the presence of an intraneural cyst were present, despite the study being read as negative (Fig. 5C and D). Four months postoperatively, the patient had recovered normal dorsiflexion.

\section{Case 6: Does Size Matter? Small Cyst, Severe Symptoms}

A 32-year-old man developed acute lateral knee pain that radiated to the great toe after lifting a sofa. The next day he developed a foot drop. EMG showed a deep peroneal predominant neuropathy. An MRI study demonstrated a complex cyst arising from the STFJ with a question- 

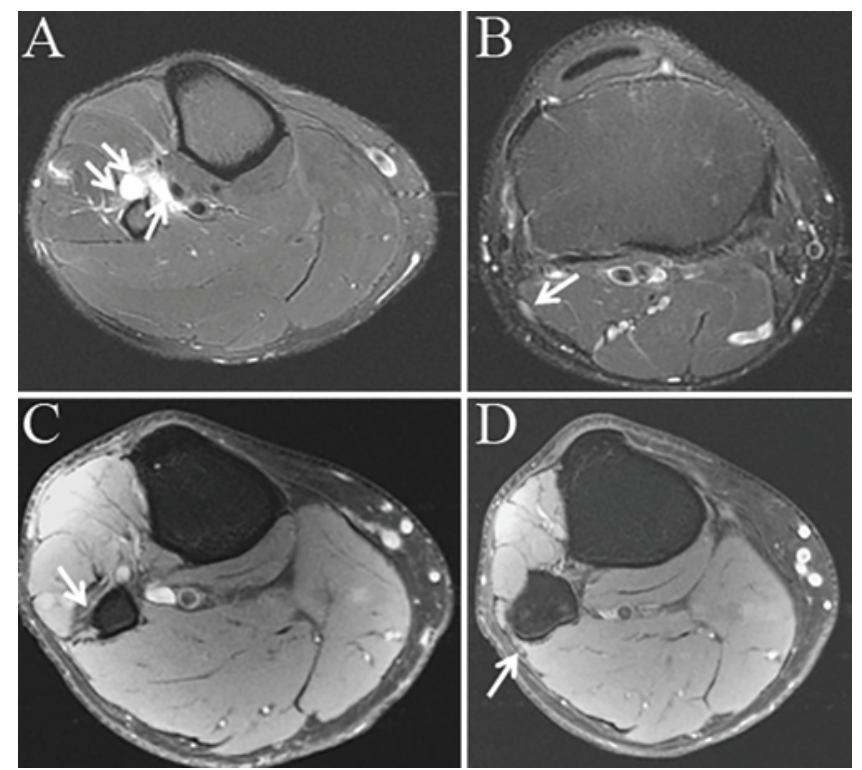

FIG. 6. Case 6. A: Axial T2-weighted image with fat suppression, showing extraneural cyst (double arrows) as well as adventitial cyst (arrow). B: Axial T2-weighted image with fat suppression, showing subtle T2 hyperintensity within the common peroneal nerve (arrow) suggestive of an intraneural cyst. C: Axial T1-weighted image obtained after injection of intraarticular gadolinium, showing contrast within the articular branch (arrow). D: Axial T1-weighted image obtained after injection of intraarticular gadolinium, showing contrast within the common peroneal nerve (arrow), consistent with an intraneural cyst.

able small peroneal intraneural component, an extraneural component, and an intravascular component (Fig. 6A and B). On our evaluation, the patient had minimal activation of the tibialis anterior muscle with only trace weakness in the peroneus muscles. Given the possibility of an intraneural component of the cyst on MRI, an MR arthrogram was ordered. The MR arthrogram demonstrated again the complex cyst and showed contrast passing from the STFJ into an intraneural component of the cyst within the common peroneal nerve and its articular branch (Fig. 6C and D). The patient did not elect to undergo surgery and has not been seen in follow-up.

\section{Discussion}

\section{The Nearly Invisible Cyst as Part of the Roller Coaster Phenomenon}

The formation of intraneural ganglion cysts is a dynamic process. Our findings demonstrate that the life cycle of an intraneural ganglion can involve a phase in which it is nearly invisible. Several snapshots in time may capture dramatic fluctuations in size and configuration, spanning the spectrum and taking on the course of a roller coaster (Fig. 7). Based on serial imaging studies, Cases 1-3 provided the perfect setting to acknowledge the entity, the nearly invisible cyst. Cases 4-6 then provided appreciation of the diagnosis of the same entity in a single snapshot but with supplementary supportive evidence. The nearly invisible cyst described here would be consistent with the "occult" intraneural cyst that our group has recently demonstrated to be isolated to the articular branch of the lateral plantar nerve in a patient who underwent surgery for presumed tarsal tunnel syndrome. ${ }^{4}$ We have previously alluded to spontaneous regression of cysts, but here we substantiate this concept and describe spontaneous regression to the point of near-complete resolution, without any evidence of cyst rupture. ${ }^{8,13}$

\section{Dynamic Cyst Morphology as Part of the Roller Coaster}

While these cases demonstrate that cyst size is dynamic, with cysts growing and shrinking over time possibly even to the point of resolution, the roller coaster phenomenon is not limited to size but also involves changes in morphology. It is not limited to the intraneural component but can also involve other compartments involved in the cyst such as the extraneural space and intravascular compartment. We have observed cases in which the intravascular compartment has shrunk to the point of complete resolution (Fig. 8). Interventions such as operative decompression or percutaneous aspiration also shift the dynamics that determine the size and morphology of the cyst such that postoperative recurrent intraneural cysts often take on a different size and morphology from the original cyst (Fig. 9). The morphology of the cyst is a result of constantly changing pressure differences within the STFJ and the compartments involved in the cyst (e.g., intraneural, intravascular, extraneural). Scar formation and

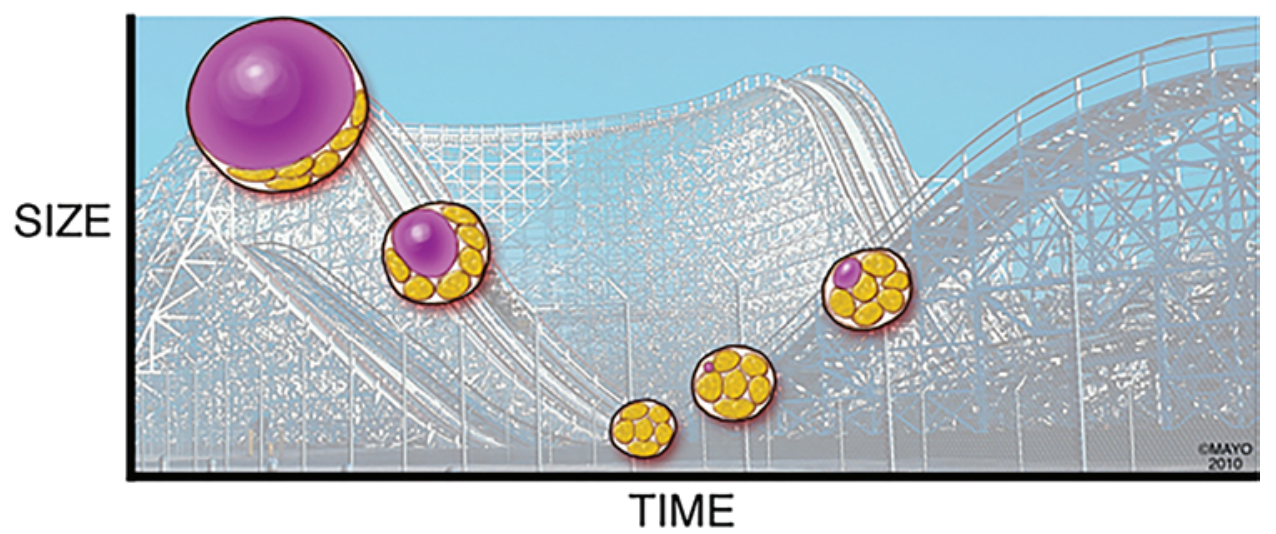

FIG. 7. Schematic diagram of the roller coaster of change in size and morphology over the life of a peroneal intraneural cyst. By permission of Mayo Foundation for Medical Education and Research. All rights reserved. 


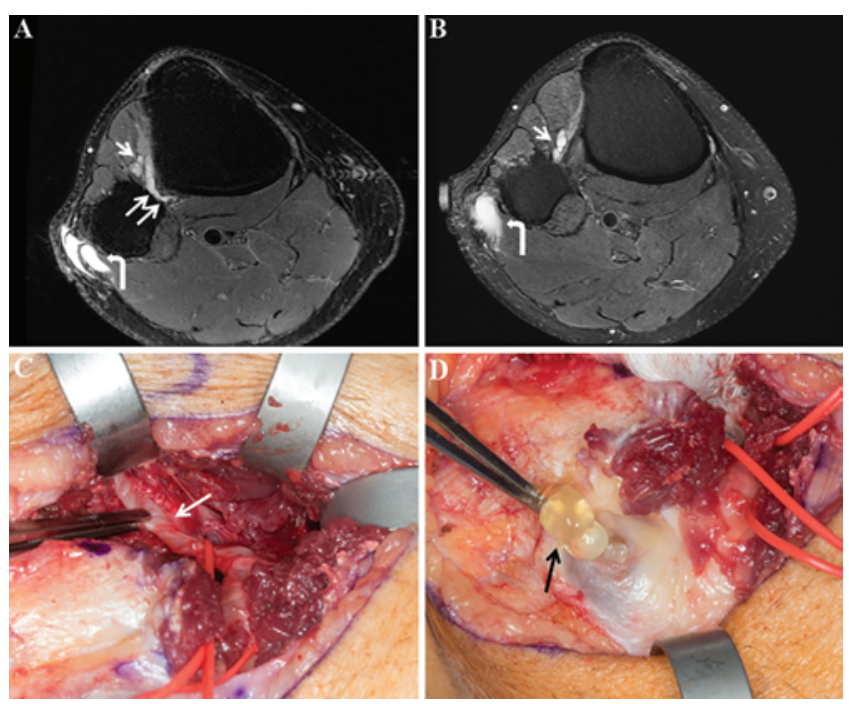

FIG. 8. A 54-year-old man presented with fluctuating symptoms of deep peroneal neuropathy. A: Initial axial T2-weighted image with fat suppression showing cyst within the common peroneal nerve (curved arrow) and tibialis anterior branch (arrow) as well as within the wall of an arterial articular branch (double arrow). B: Because of a delay in referral, the subsequent axial T2-weighted image with fat suppression was obtained 1 year later. Cyst is visible within the common peroneal nerve (curved arrow) and tibialis anterior branch (arrow). The intravascular component of the cyst was no longer present. C: Intraoperative photograph showing a cystic articular branch (arrow). D: Intraoperative photograph showing synovial fluid (arrow) released through a small opening in the cyst wall.

inflammation generated by interventions and locoregional changes within the involved compartments, such as blood pressure for the intravascular compartment, potentially alter these pressure dynamics, allowing for alterations in size and morphology.

\section{The Nearly Invisible Cyst: The Tip of the Iceberg}

While once thought to be uncommon, peroneal intra- neural ganglion cysts are increasingly being recognized as an important cause of peroneal neuropathy, occurring in up to $18 \%$ of cases.22 Given the number of patients we see with "negative" MRI findings who turn out to have nearly invisible cysts, even this may be underestimating the true frequency. We believe that a subgroup of patients of unknown size with peroneal neuropathy have unrecognized (nearly invisible) intraneural cysts.

\section{An Explanation for "Negative" MRI or Ultrasound Findings}

All of our cases had very subtle imaging findings, which were or could have been easily missed (explaining "negative" MRI or ultrasound findings), unless the suspicion was there a priori. Cases 1 and 2 clearly demonstrated that intraneural ganglion cysts can shrink dramatically, and, at times, rapidly. MR arthrography can be useful when subtle signs of the presence of an intraneural cyst are not completely convincing to demonstrate the passage of intraarticular contrast into the articular branch and confirm the presence of an intraneural cyst, as in Cases 2, 4, and 6., 5,12,19 The articular branch of the tibial nerve in these studies serves as an internal control. Intraarticular contrast was not seen within this branch in these studies, confirming that passage of the contrast into the peroneal articular branch was pathologic. Imaging may be performed when the cyst happens to be at the nadir of the roller coaster, with only easy to miss, subtle signs on imaging. Particularly if the slice thickness is thick, the findings could be missed altogether. Experienced radiologists using optimal techniques and high-resolution imaging will improve the recognition of these nearly invisible cysts.

\section{Clues to the Presence of an Intraneural Cyst}

Additional clinical and imaging clues can be helpful in identifying nearly invisible cysts, even in cases of "negative" MRI findings. In Cases 2 and 3, the obvious presence of a cyst on ultrasound clued us in to examining the MRI studies in detail for signs of a cyst. However, we believe a number of other clinical and radiological findings can
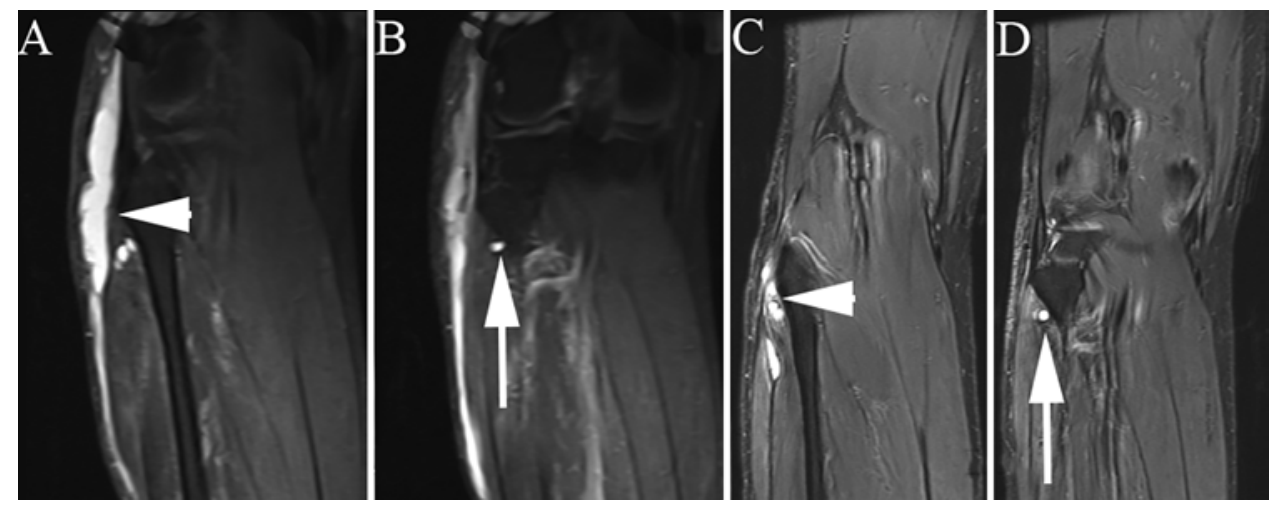

FIG. 9. A 49-year-old man presented with a complete foot drop and pain radiating from the knee to the great toe. MRI was performed (A and B). He underwent operative decompression of the common peroneal nerve and aspiration of the cyst at an outside institution. His cyst subsequently recurred and additional MRI was performed 2 months postoperatively (C and D). A and B: Coronal T2-weighted images with fat suppression showing cyst, within the common peroneal nerve (arrowhead, A) and within the articular branch (arrow, B). C and D: The cyst recurred. Two months postoperatively, coronal T2-weighted images with fat suppression were obtained, showing cyst within the common peroneal nerve (arrowhead) with a loculated appearance and morphology differing from the initial cyst (C) and cyst within the articular branch (arrow, D). 
be clues to the presence of an intraneural cyst, prompting in-depth review of the imaging studies. First, a number of these cases illustrate what we have anecdotally observed: peroneal intraneural cysts often present with deep peroneal predominance. Fluctuating symptoms, such as seen in Case 4, also seem to correlate with the presence of a cyst. $^{23}$ From an imaging standpoint, the deep peroneal predominance can be observed with subacute denervation changes in the tibialis anterior muscle, such as in Cases 2, 3 , and 5. The joint of origin is the STFJ, so imaging abnormalities in this joint should also prompt consideration of a cyst. Degenerative changes, joint edema, and intraosseous ganglion cysts, as seen in Cases 4 and 5, suggest an abnormal joint that may be at risk for capsular defects and cyst formation. These imaging findings are clues to scrutinize the articular branch and peroneal nerve for subtle evidence of an intraneural cyst. Frequently, when subtle cyst is observed, it is located only in the anterior portion of the common peroneal nerve, often at around the 12 o'clock position (Figs. 2 and 3). The internal topography of the common peroneal nerve has been described..$^{2,21}$ The fascicles of the articular branch run with the fascicles of the deep peroneal nerve in the anterolateral portion of the common peroneal nerve at the level of the fibular head. This is the place to look on MRI. The "clock face" configuration that we have previously described still applies. ${ }^{20}$ On both MRI and MR arthrography, the critical positions to look for abnormalities correspond to locations about the clock face where the signet ring, transverse limb, and tail signs appear. In the case of nearly invisible cysts, these signs may not be fully developed, but cyst is present in those topographical locations. Understanding the "critical times" around the clock face often allows one to recognize a number of the important findings that we have described, all in a single MRI slice (Fig. 10).

\section{Subtle or Even Negative Pathology}

In Case 5, we demonstrate the subtle histological changes that can be seen, despite the articular branch being clearly cystic and enlarged at surgery. Despite imaging evidence of a clear intraneural cyst in Case 2, findings from the histopathological analysis of the articular branch specimen were negative. In truth, we believe that the full spectrum of an intraneural ganglion cyst can include negative histological findings that do not reveal cyst in the articular branch. A number of explanations can be postulated for this. It is plausible that the fluid track from its joint origin to the parent nerve is a potential space that allows passage of fluid and then shuts down. The articular branch might not always harbor a true cyst but rather might simply be a passageway. In other instances, a true cyst may form within the articular branch. Finding no evidence of a cyst might represent a sampling error. A true cyst might not be present throughout the entire articular branch, and the portion submitted for review might not contain cyst. Loss of cyst fluid may occur from the operative intervention or from the processing and handling of the specimen.

\section{Does the Identification of Small Intraneural Cysts Matter?}

The roller coaster of intraneural ganglion cysts has a number of implications. Does size matter relative to
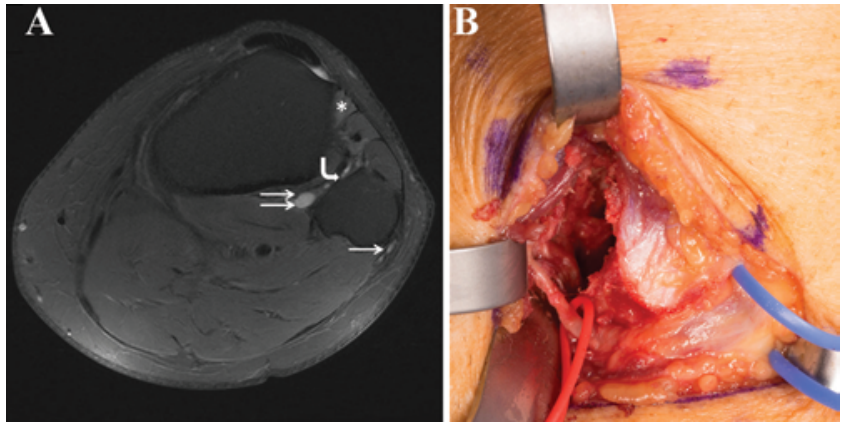

FIG. 10. A 49-year-old man presented with a complete foot drop and numbness in the first dorsal web space. A: Axial T2-weighted MR image with fat suppression demonstrating subtle intraneural cyst (arrow) within the common peroneal nerve at the typical location of the signet ring sign (4-5 o'clock) and within the articular branch (curved arrow) at the typical location of the tail sign (11-12 o'clock). Extraneural cyst (double arrow) and subacute denervation changes in the tibialis anterior (asterisk) are also noted on this slice. Subtle cyst extended to the level of the sciatic nerve bifurcation in the anterior-most portion of the common peroneal nerve (not shown). B: To our surprise, at the time of surgery 7 weeks after the MR image was obtained, the intraneural cyst in the common peroneal nerve (blue loop) and in the articular branch (red loop) had significantly increased in size compared with what was observed on the image.

symptoms? We have observed a number of cases similar to Case 6, where small cysts are associated with severe neurological symptoms. One could interpret this as size not being a determinant in the severity of symptoms. However, it could be that the imaging study available captured the cyst at its nadir, while at its peak it was (and perhaps will be again) a large cyst. So, maybe it is that maximum size in the life of the cyst better correlates with symptoms. Alternatively, it may be that size does not matter relative to symptoms. This question remains unanswered. Small, nearly invisible, unrecognized cysts may contribute to peroneal neuropathy in a significant subset of patients and may be a possible cause of operative treatment failure in a subset of patients.

\section{Limitations}

The main limitation to this study is the cohort size. Each point is illustrated through a single case. A single case, however, is enough to prove the concepts proposed. What remains to be shown is the frequency at which each phenomenon occurs. Each case helps answer questions but also raises questions that should be systematically studied through larger investigations.

While these examples help establish reasons that intraneural ganglion cysts may be underrecognized and often missed and provide clues that may help increase recognition, the study does not address treatment recommendations. When a cyst is identified preoperatively, we typically perform a peroneal nerve decompression with resection of both the articular branch and STFJ, but in several examples provided when the cyst was nearly invisible, we performed only resection of the articular branch without resection of the STFJ. When this option should be exercised is unclear. The pathology in these small cysts may still occur at the entrance to the fibular tunnel. The nerve 
may be slightly enlarged and, as a result, compressed by the deep fascia of the peroneus longus muscle. Decompression may be enough to resolve the symptoms despite not specifically addressing the cyst and source of the pathology. Additional work needs to be done to understand what recognition of nearly invisible cysts means for treatment and outcomes.

\section{Conclusions}

We demonstrate here that peroneal intraneural ganglion cysts ride a roller coaster of change in both size and morphology over time, and we introduce the nearly invisible cyst as one end of the spectrum. The fact that almost invisible cysts exist and are frequently missed on MRI means that intraneural ganglion cysts are likely an underrecognized cause of peroneal neuropathy. Clues to the presence of an intraneural cyst that we have identified may help increase the recognition of nearly invisible cysts.

\section{References}

1. Desy NM, Wang H, Elshiekh MA, Tanaka S, Choi TW, Howe $\mathrm{BM}$, et al: Intraneural ganglion cysts: a systematic review and reinterpretation of the world's literature. J Neurosurg 125:615-630, 2016

2. Gustafson KJ, Grinberg Y, Joseph S, Triolo RJ: Human distal sciatic nerve fascicular anatomy: implications for ankle control using nerve-cuff electrodes. J Rehabil Res Dev 49:309-321, 2012

3. Lipinski LJ, Rock MG, Spinner RJ: Peroneal intraneural ganglion cysts at the fibular neck: the layered "U" surgical approach to the articular branch and superior tibiofibular joint. Acta Neurochir (Wien) 157:837-840, 2015

4. Prasad N, Amrami KK, Yangi K, Spinner RJ: Occult isolated articular branch cyst of the lateral plantar nerve. J Foot Ankle Surg 56:78-81, 2017

5. Puffer RC, Spinner RJ, Amrami KK, Skinner JA: Direct superior tibiofibular joint arthrography demonstrates consistent communication with the tibiofemoral joint. Clin Anat 27:1128-1129, 2014

6. Puffer RC, Spinner RJ, Murthy NS, Amrami KK: CT and MR arthrograms demonstrate a consistent communication between the tibiofemoral and superior tibiofibular joints. Clin Anat 26:253-257, 2013

7. Scherman BM, Bilbao JM, Hudson AR, Briggs SJ: Intraneural ganglion: a case report with electron microscopic observations. Neurosurgery 8:487-490, 1981

8. Shahid KR, Hébert-Blouin MN, Amrami KK, Spinner RJ: Extraneural rupture of intraneural ganglion cysts. J Surg Orthop Adv 20:136-141, 2011

9. Spinner RJ, Amrami KK: Intraneural ganglion of the suprascapular nerve: case report. J Hand Surg Am 31:1698-1699, 2006

10. Spinner RJ, Amrami KK, Angius D, Wang H, Carmichael SW: Peroneal and tibial intraneural ganglia: correlation between intraepineurial compartments observed on magnetic resonance images and the potential importance of these compartments. Neurosurg Focus 22(6):E17, 2007

11. Spinner RJ, Amrami KK, Kliot M, Johnston SP, Casañas J: Suprascapular intraneural ganglia and glenohumeral joint connections. J Neurosurg 104:551-557, 2006
12. Spinner RJ, Amrami KK, Rock MG: The use of MR arthrography to document an occult joint communication in a recurrent peroneal intraneural ganglion. Skeletal Radiol 35:172-179, 2006

13. Spinner RJ, Amrami KK, Wolanskyj AP, Desy NM, Wang H, Benarroch EE, et al: Dynamic phases of peroneal and tibial intraneural ganglia formation: a new dimension added to the unifying articular theory. J Neurosurg 107:296-307, 2007

14. Spinner RJ, Atkinson JL, Scheithauer BW, Rock MG, Birch R, Kim TA, et al: Peroneal intraneural ganglia: the importance of the articular branch. Clinical series. J Neurosurg 99:319-329, 2003

15. Spinner RJ, Atkinson JL, Tiel RL: Peroneal intraneural ganglia: the importance of the articular branch. A unifying theory. J Neurosurg 99:330-343, 2003

16. Spinner RJ, Desy NM, Amrami KK: Sequential tibial and peroneal intraneural ganglia arising from the superior tibiofibular joint. Skeletal Radiol 37:79-84, 2008

17. Spinner RJ, Desy NM, Amrami KK: The unifying articular (synovial) origin for intraneural ganglion cysts: moving beyond a theory. J Hand Surg Am 41:e223-e224, 2016

18. Spinner RJ, Hébert-Blouin MN, Rock MG, Amrami KK: Extreme intraneural ganglion cysts. J Neurosurg 114:217-224, 2011

19. Spinner RJ, Hébert-Blouin MN, Skinner JA, Amrami KK: Knee MR arthrographic proof of an articular origin for combined intraneural and adventitial cysts. Acta Neurochir (Wien) 152:919-923, 2010

20. Spinner RJ, Luthra G, Desy NM, Anderson ML, Amrami $\mathrm{KK}$ : The clock face guide to peroneal intraneural ganglia: critical "times" and sites for accurate diagnosis. Skeletal Radiol 37:1091-1099, 2008

21. Sunderland S, Ray LJ: The intraneural topography of the sciatic nerve and its popliteal divisions in man. Brain 71:242-273, 1948

22. Visser LH: High-resolution sonography of the common peroneal nerve: detection of intraneural ganglia. Neurology 67:1473-1475, 2006

23. Young NP, Sorenson EJ, Spinner RJ, Daube JR: Clinical and electrodiagnostic correlates of peroneal intraneural ganglia. Neurology 72:447-452, 2009

\section{Disclosures}

The authors report no conflict of interest concerning the materials or methods used in this study or the findings specified in this paper.

\section{Author Contributions}

Conception and design: Spinner, Wilson, Hébert-Blouin, Amrami. Acquisition of data: Spinner, Wilson, Hébert-Blouin. Analysis and interpretation of data: all authors. Drafting the article: Spinner, Wilson. Critically revising the article: all authors. Reviewed submitted version of manuscript: all authors. Approved the final version of the manuscript on behalf of all authors: Spinner. Study supervision: Spinner.

\section{Correspondence}

Robert J. Spinner, Department of Neurosurgery, Mayo Clinic, 200 1st St. SW, Rochester, MN 55905. email: spinner.robert@ mayo.edu. 